\title{
Sharp estimates for bubbling solutions of a fourth order mean field equation
}

\author{
CHANG-SHOU LIN AND JUNCHENG WEI
}

Abstract. We consider a sequence of multi-bubble solutions $u_{k}$ of the following fourth order equation

$$
\Delta^{2} u_{k}=\rho_{k} \frac{h(x) e^{u_{k}}}{\int_{\Omega} h e^{u_{k}}} \text { in } \Omega, \quad u_{k}=\Delta u_{k}=0 \text { on } \partial \Omega,
$$

where $h$ is a $C^{2, \beta}$ positive function, $\Omega$ is a bounded and smooth domain in $\mathbb{R}^{4}$, and $\rho_{k}$ is a constant such that $\rho_{k} \leq C$. We show that (after extracting a subsequence), $\lim _{k \rightarrow+\infty} \rho_{k}=32 \sigma_{3} m$ for some positive integer $m \geq 1$, where $\sigma_{3}$ is the area of the unit sphere in $\mathbb{R}^{4}$. Furthermore, we obtain the following sharp estimates for $\rho_{k}$ :

$$
\begin{aligned}
\rho_{k}-32 \sigma_{3} m= & c_{0} \sum_{j=1}^{m} \epsilon_{k, j}^{2}\left(\sum_{l \neq j} \Delta G_{4}\left(p_{j}, p_{l}\right)+\Delta R_{4}\left(p_{j}, p_{j}\right)+\frac{1}{32 \sigma_{3}} \Delta \log h\left(p_{j}\right)\right) \\
& +o\left(\sum_{j=1}^{m} \epsilon_{k, j}^{2}\right)
\end{aligned}
$$

where $c_{0}>0, \log \frac{64}{\epsilon_{k, j}^{4}}=\max _{x \in B_{\delta}\left(p_{j}\right)} u_{k}(x)-\log \left(\int_{\Omega} h e^{u_{k}}\right)$ and $u_{k} \rightarrow 32 \sigma_{3} \sum_{j=1}^{m} G_{4}\left(\cdot, p_{j}\right)$ in $C_{\mathrm{loc}}^{4}\left(\Omega \backslash\left\{p_{1}, \ldots, p_{m}\right\}\right)$.

This yields a bound of solutions as $\rho_{k}$ converges to $32 \sigma_{3} m$ from below provided that

$$
\sum_{j=1}^{m}\left(\sum_{l \neq j} \Delta G_{4}\left(p_{j}, p_{l}\right)+\Delta R_{4}\left(p_{j}, p_{j}\right)+\frac{1}{32 \sigma_{3}} \Delta \log h\left(p_{j}\right)\right)>0 .
$$

The analytic work of this paper is the first step toward computing the LeraySchauder degree of solutions of equation $(*)$.

Mathematics Subject Classification (2000): 35B40 (primary); 35B45, 35J40 (secondary).

Received December 12, 2006; accepted in revised form November 13, 2007. 


\section{Introduction}

In this paper, we initiate the study of the following fourth order mean field equation

$$
\begin{cases}\Delta^{2} u=\rho \frac{h e^{u}}{\int_{\Omega} h e^{u}} & \text { in } \Omega, \\ u=\Delta u=0 & \text { on } \partial \Omega .\end{cases}
$$

This is the first of a series of two papers on computing the Leray-Schauder degree for solutions of (1.1). In this first paper, we compute the sharp estimates of the bubbling rate of multiple bubble solutions.

In dimension two, the analogous problem

$$
\begin{cases}-\Delta u=\rho \frac{h e^{u}}{\int_{\Omega} h e^{u}} & \text { in } \Omega \\ u=0 & \text { on } \partial \Omega\end{cases}
$$

where $\Omega$ is a smooth and bounded domain in $\mathbb{R}^{2}$, has been extensively studied by many authors. We summarize the results for (1.2) and identify the difficulty in studying (1.1) now. Let $\left(u_{k}, \rho_{k}\right)$ be a bubbling sequence to (1.2) with $\rho_{k} \leq$ $C, \max _{x \in \Omega} u_{k}(x) \rightarrow+\infty$. Then it has been proved that

(P1) (no boundary bubbles) $u_{k}$ is uniformally bounded near a neighborhood of $\partial \Omega$ (Ma-Wei [18]);

(P2) (bubbles are simple) $\rho_{k} \rightarrow 8 m \pi$ for some $m \geq 1$ and $u_{k}(x) \rightarrow 8 \pi \sum_{j=1}^{m} G_{2}\left(\cdot, p_{j}\right)$ in $C^{2}\left(\Omega \backslash\left\{p_{1}, \ldots, p_{m}\right\}\right)[2,13,18,21]$, where $G_{2}$ is the Green function of $-\Delta$ with Dirichlet boundary condition;

(P3) (sup + inf estimates) at each bubble $p_{k, j}$ where $u_{k}\left(p_{k, j}\right)=\max _{x \in B_{\delta}\left(p_{j}\right)} u_{k}(x)$, the following refined estimates hold $[5,12,13]$

$$
\left|u_{k}(x)-u_{k}\left(p_{k, j}\right)-\log \frac{1}{\left(1+\frac{\left|x-x_{k, j}\right|^{2}}{\epsilon_{k, j}^{2}}\right)^{2}}\right| \leq C
$$

where $u_{k}\left(p_{k, j}\right)-\log \left(\int_{\Omega} h e^{u_{k}}\right)=\log \frac{1}{\epsilon_{k, j}^{2}} ;$

(P4) (exact bubbling rate) It holds then [7]

$$
\rho_{k}-8 m \pi=c_{0} \sum_{j=1}^{m} h\left(p_{k, j}\right)^{-1} \Delta \log h\left(p_{k, j}\right) \epsilon_{k, j}^{2} \log \frac{1}{\epsilon_{k, j}}+O\left(\sum_{j=1}^{m} \epsilon_{k, j}^{2}\right) ;
$$

(P5) (Leray-Schauder degree) Li [12] initiated the program of computing the Leray-Schauder degree of solutions to (1.2). He showed that the Leray-Schauder 
degree remains a constant for $\rho \in(8 \pi(m-1), 8 \pi m)$ and that the degree depends only on the genus of $\Omega$. Chen and Lin [8] obtained the exact degree counting formula as follows:

$$
d(\rho)= \begin{cases}\frac{1}{m !}(-\chi(\Omega)+1) \ldots(-\chi(\Omega)+m) & \text { for } m>0 \\ 1 & \text { for } m=0\end{cases}
$$

where $\chi(\Omega)$ is the Euler characteristic of $\Omega$.

In this and subsequent paper [17], we carry out the same program for equation (1.1). It will be shown that $d(\rho)$-the Leray-Schauder degree of (1.1) can be defined as long as $\rho \neq 32 m \sigma_{3}$, where $\sigma_{3}$ is the area of unit sphere in $\mathbb{R}^{4}$. The main purpose of this paper and the subsequent one [17] is to compute $d(\rho)$. In these two papers, we prove, among other things, the following theorem:

Theorem A. Let $32 m \sigma_{3}<\rho<32(m+1) \sigma_{3}$ and $d(\rho)$ be the Leray-Schauder degree for equation (1.1). Then

$$
d(\rho)= \begin{cases}\frac{1}{m !}(-\chi(\Omega)+1) \ldots(-\chi(\Omega)+m) & \text { for } m>0 \\ 1 & \text { for } m=0\end{cases}
$$

where $\chi(\Omega)$ is the Euler characteristic of $\Omega$.

Remark 1.1. We are informed by Prof. Malchiodi that he obtained a similar degree counting formula for the corresponding prescribing $Q$-curvature problem on a four dimensional compact manifold, [20]. He used a different approach -the Morse theory approach- to obtain the formula. We remark that on compact manifolds, one does not need to prove property (P1). On the other hand, one of the main difficulties in our proof is the property $(\mathrm{P} 1)$.

As a consequence of Theorem A, equation (1.1) always possesses a solution for $\rho \neq 32 m \sigma_{3}$ whenever the Euler characteristic $\chi(\Omega) \leq 0$. (Here $m$ can be made $\geq 2$, by results of Lin-Wei [16].) On the other paper, when $\chi(\Omega)>0$, the situation is much different than the second order case. For example, when $\Omega$ is a ball, we can prove the existence of at least one solution when $\rho \in\left(0,64 \pi \sigma_{3}\right)$. See the remark after Corollary 1.3. The complete proof of Theorem A will be given in [17], the second part of this series of papers.

Set $d_{m}^{+}=\lim _{\rho \rightarrow 8 m \pi+} d(\rho)$ and $d_{m}^{-}=\lim _{\rho \rightarrow 32 m \sigma_{3}} d(\rho)$. One of the main steps in the proof of Theorem A is to calculate the gap $d_{m}^{+}-d_{m}^{-}$for any integer $m \geq 1$. Once this is known, $d(\rho)$ can be computed inductively on $m$. Clearly, the gap of $d_{m}^{+}-d_{m}^{-}$is due to the occurrence of blowup solutions when $\rho \rightarrow 32 m \sigma_{3}$. Thus an important question is to analyze the blowup behavior of sequence of solutions $u_{k}$ to (1.1) and to know the signs $\rho_{k}-32 m \sigma_{3}$.

In this paper, we shall obtain estimates analogous to (1.4) for bubbling solutions to (1.1). To this end, we have to first resolve the analogous properties (P1), 
(P2) and (P3) for problem (1.1). Once we obtain (P1), (P2) follows from results in Wei [23]. So we just need to prove (P1) and (P3). Here the problem arises since the method of Kelvin transform in obtaining (P1) and the method of moving spheres in obtaining (P3) seem not applicable for (1.1). We overcome these difficulties by using various new techniques. (After we obtain (P1)-(P3), the Leray-Schauder degree $d(\rho)$ of (1.1) for $\rho \neq 32 m \sigma_{3}$ can be well-defined.)

The following is the main result of this paper:

Theorem 1.2. Let $h$ be a positive $C^{2, \beta}$ function in $\Omega$ and $u_{k}$ be a sequence of blowup solutions of (1.1) with $\rho=\rho_{k}$. Then (after extracting a subsequence), $\lim _{k \rightarrow+\infty} \rho_{k}=32 \sigma_{3} m$ for some positive integer $m$. Furthermore,

$$
\begin{aligned}
& \rho_{k}-32 \sigma_{3} m \\
& =c_{0} \sum_{j=1}^{m}\left(h\left(p_{k, j}\right)\right)^{-\frac{1}{2}} \epsilon_{k, j}^{2}\left[\frac{1}{32 \sigma_{3}} \Delta \log h\left(p_{k, j}\right)+\Delta R_{4}\left(p_{k, j}, p_{k, j}\right)\right. \\
& \left.+\sum_{i \neq j} \Delta G_{4}\left(p_{k, j}, p_{k, i}\right)\right] \\
& +o\left(\sum_{j=1}^{m} \epsilon_{k, j}^{2}\right)
\end{aligned}
$$

where $c_{0}>0$ is a generic constant, $G_{4}(\cdot, P)$ is the Green function of $\Delta^{2}$ with Navier boundary condition $u=\Delta u=0$ on $\partial \Omega, R_{4}$ is the regular part of $G_{4}$, $p_{k, j}$ are the local maximum points of $u_{k}$ on $B_{\delta}\left(p_{j}\right)$, and $\log \frac{64}{\epsilon_{k, j}^{4}}=u_{k}\left(p_{k, j}\right)-$ $\log \left(\int_{\Omega} h e^{u_{k}}\right)$.

Clearly Theorem 1.2 implies the following:

Corollary 1.3. Let $h(x)$ be a $C^{2, \beta}$ positive function and satisfy

$$
\sum_{j=1}^{m}\left(h\left(p_{j}\right)\right)^{-\frac{1}{2}}\left[\frac{1}{32 \sigma_{3}} \Delta \log h\left(p_{j}\right)+\Delta R_{4}\left(p_{j}, p_{j}\right)+\sum_{l \neq j} \Delta G_{4}\left(p_{l}, p_{j}\right)\right]>0
$$

for all $\left(p_{1}, \ldots, p_{m}\right)$ satisfying

$$
\nabla\left(\frac{1}{32 \sigma_{3}} \log h\left(p_{j}\right)+R_{4}\left(p_{j}, p_{j}\right)+\sum_{l \neq j} G_{4}\left(p_{l}, p_{j}\right)\right)=0, j=1, \ldots, m .
$$

Then for any compact interval $I \subset\left(32 \sigma_{3}(m-1), 32 \sigma_{3} m\right]$, there exists a constant $C>0$ such that

$$
u(x) \leq C \text { for } x \in \Omega
$$

for any solution $u$ of (1.1) with $\rho \in I$.

As a consequence, if $\Delta h(x) \geq 0$, then (1.10) holds for any solution $u$ of (1.1) with $\rho \in\left(0,32 \sigma_{3}\right]$. 
Remark 1.4. 1. Corollary 1.3 extends earlier results of Lin and Wei [16] where we proved Corollary 1.3 for $m=1, h=1$. We note that when $\Omega=B_{1}$ and $h(x)=1,(1.2)$ has no solution when $\rho \geq 8 \pi$. However, for (1.1), a solution always exists when $\rho \leq 32 \sigma_{3}$ [16]. On the other hand, a solution with a single bubble has been constructed in [3]. By Theorem 1.2, since $\Delta R_{4}\left(p_{j}, p_{j}\right)>0$, this shows that problem (1.1) has a solution for $\rho>32 \sigma_{3}$ and $\left|\rho-32 \sigma_{3}\right|$ small. By Theorem A, $d(\rho)=0$ for $\rho \in\left(32 \sigma_{3}, 64 \sigma_{3}\right)$. Thus when $\Omega=B_{1}$ and $h=1$, problem (1.1) has a solution for $\rho \in\left(0,64 \sigma_{3}\right)$. In fact, we conjecture that a solution to (1.1) exists for any $\rho>0$.

2. Theorem 1.1 can be extended easily to the following $n$-th order mean field type equation

$$
\begin{cases}(-\Delta)^{n} u=\rho \frac{h e^{u}}{\int_{\Omega} h e^{u}} & \text { in } \Omega, \\ (-\Delta)^{j} u=0 & \text { on } \partial \Omega, j=0, \ldots, n-1\end{cases}
$$

where $\Omega$ is a smooth and bounded domain in $\mathbb{R}^{2 n}$. In particular, we have the same degree counting formula for solutions to (1.11)

$$
d(\rho)= \begin{cases}\frac{1}{m !}(-\chi(\Omega)+1) \cdots(-\chi(\Omega)+m) & \text { for } m>0 \\ 1 & \text { for } m=0\end{cases}
$$

where $\rho \in\left(m 2^{2 n}(n-1) ! n ! \sigma_{2 n-1},(m+1) 2^{2 n}(n-1) ! n ! \sigma_{2 n-1}\right)$. This then implies that (1.11) always has a solution if $\rho \neq m 2^{2 n}(n-1) ! n ! \sigma_{2 n-1}$ and $\chi(\Omega) \leq 0$.

Semilinear equations involving exponential nonlinearity and fourth order elliptic operator appear naturally in conformal geometry and in particular in prescribing $Q$-curvature on 4-dimensional Riemannian manifold $M$ (see e.g. Chang-Yang [6])

$$
P_{g} w+2 Q_{g}=2 \tilde{Q}_{g_{w}} e^{4 w}
$$

where $P_{g}$ is the so-called Paneitz operator:

$$
P_{g}=\left(\Delta_{g}\right)^{2}+\delta\left(\frac{2}{3} R_{g} I-2 \operatorname{Ric}_{g}\right) d,
$$

$g_{w}=e^{2 w} g, Q_{g}$ is $Q$-curvature under the metric $g$, and $\tilde{Q}_{g_{w}}$ is the $Q$-curvature under the new metric $g_{w}$.

Integrating (1.12) over M, we obtain

$$
k_{g}:=\int_{M} Q_{g}=\int_{M}\left(\tilde{Q}_{g_{w}}\right) e^{4 w}
$$

where $k_{g}$ is conformally-invariant. Thus, we can write (1.12) as

$$
P_{g} w+2 Q_{g}=k_{g} \frac{\tilde{Q}_{g_{w}} e^{4 w}}{\int_{M} \tilde{Q}_{g_{w}} e^{4 w}}
$$


In the special case, where the manifold is the Euclidean space, $P_{g}=\Delta^{2}$, and (1.13) becomes

$$
\Delta^{2} w=\rho \frac{h(x) e^{4 w}}{\int_{\Omega} h(x) e^{4 w}} .
$$

There is now an extensive literature about this problem, we refer to AdimurthiRobert-Struwe [1], Baraket-Dammak-Ouni-Pacard [3], Druet-Robert [9], HebeyRobert [10], Hebey-Robert-Wen [11], Malchiodi [19] and the references therein.

The organization of this paper is as follows: The statements for properties (P1)-(P3) are collected in Section 2 where important preliminaries are presented. The proof of (P1) is given in the Appendix A and the proof of (P3) is given in Section 3. Finally in Section 4, we prove Theorem 1.2. Though we essentially follow those of [7], we simplify and give a new proof of the key estimates-Estimate $\mathrm{C}$ in Section 5.

Throughout this paper, unless otherwise stated, the letter $C$ will always denote various generic constants which are independent of $k \geq 1$.

ACKNOWLEDGEMENTS. The research of the first author is partially supported by a research Grant from NSC of Taiwan. The research of the second author is partially supported by an Earmarked Grant from RGC of Hong Kong.

\section{Preliminaries}

We begin with the following lemma which excludes the boundary blowups. The proof of it is by adopting the method used in our previous paper [16] and is given in Appendix A.

Lemma 2.1. Let $u$ be a solution to (1.1) with $\rho \leq C$. Then there exists $a \delta>0$ such that $u(x) \leq C$ for all $x$ such that $d(x, \partial \Omega) \leq \delta$.

Let $G_{4}$ denote the Green's function of $\Delta^{2}$ under the Navier boundary condition, that is

$$
\Delta^{2} G_{4}(x, y)=\delta(x-y),\left.G_{4}\right|_{\partial \Omega}=\left.\Delta G_{4}\right|_{\partial \Omega}=0 .
$$

We decompose

$$
G_{4}(x, y)=\frac{1}{4 \sigma_{3}} \log \frac{1}{|x-y|}+R_{4}(x, y) .
$$

It is easy to see that

$$
\Delta_{x} G_{4}(x, y)<0, \Delta_{x} R_{4}(x, y)>0 .
$$

From Lemma 2.1, we derive the following lemma, whose proof follows exactly those in Wei [23] and thus omitted. 
Lemma 2.2. Let $u_{k}$ be a bubbling sequence with $\rho_{k} \leq C$. Then (after extracting a subsequence), $\rho_{k} \rightarrow 32 \sigma_{3} m$ and $u_{k}(x) \rightarrow 32 \sigma_{3} \sum_{j=1}^{m} G_{4}\left(\cdot, p_{j}\right)$, where $\left(p_{1}, \ldots, p_{m}\right)$ satisfies

$$
\nabla\left(\frac{1}{32 \sigma_{3}} \log h\left(p_{i}\right)+R_{4}\left(p_{i}, p_{i}\right)+\sum_{j \neq i} G_{4}\left(p_{i}, p_{j}\right)\right)=0, i=1, \ldots, m .
$$

We also need to recall the well-known Pohozaev's identity for solutions of fourthorder equation

$$
\Delta^{2} u=h(x) e^{u} \text { in } D
$$

We have:

Lemma 2.3. Let $u$ satisfy $\Delta^{2} u=h(x) e^{u}$ in $D$, where $D$ is a smooth and bounded domain in $\mathbb{R}^{4}$. Then we have

$$
\begin{aligned}
& \int_{D}(4 h+<x, \nabla h>) e^{u}=\int_{\partial D}<x, v>h(x) e^{u} \\
& +\int_{\partial D}\left[\frac{1}{2}|\Delta u|^{2}<x, v>-2 \frac{\partial u}{\partial v} \Delta u-<x, \nabla u>\frac{\partial \Delta u}{\partial v}-<x, \nabla \Delta u>\frac{\partial u}{\partial v}\right. \\
& +<x, v><\nabla u, \nabla \Delta u>]
\end{aligned}
$$

and for any $\xi \in \mathbb{R}^{4}$,

$$
\begin{gathered}
\int_{D}(<\xi, \nabla h>) e^{u}=\int_{\partial D} h(x) e^{u}<\xi, v> \\
\int_{\partial D}\left[\frac{1}{2}|\Delta u|^{2}<\xi, v>-<\xi, \nabla u>\frac{\partial \Delta u}{\partial v}-<\xi, \nabla \Delta u>\frac{\partial \Delta u}{\partial v}\right. \\
+<\xi, v><\nabla u, \nabla \Delta u>]
\end{gathered}
$$

Proof. In fact, multiplying $\Delta^{2} u=h(x) e^{u}$ by $x \cdot \nabla u$ and integrating by parts, we obtain the lemma.

Let $\delta_{0}$ be a fixed small constant and $u_{k}\left(p_{k, j}\right)=\max _{x \in B_{\delta}\left(p_{j}\right)} u_{k}(x)$ and

$$
e^{-c_{k}}=\frac{1}{\int_{\Omega} h(x) e^{u_{k}}}
$$

Then $c_{k} \rightarrow+\infty$ as $k \rightarrow+\infty$. Let us define

$$
l_{k, j}=u_{k}\left(p_{k, j}\right)-c_{k}, \quad e^{-\frac{l_{k, j}}{4}}=\frac{\epsilon_{k, j}}{\alpha_{4}^{\frac{1}{4}}}, \text { where } \alpha_{4}=64
$$


and

$$
l_{k}=\max _{1 \leq j \leq m} l_{k, j}, \quad \epsilon_{k}=\min _{1 \leq j \leq m} \epsilon_{k, j} .
$$

Note that $l_{k, j} \rightarrow+\infty$, as otherwise $u_{k}$ satisfies $\left|\Delta^{2} u_{k}\right| \leq C$ in $B_{\delta_{0}}\left(p_{k, j}\right), u_{k}+$ $\left|\Delta u_{k}\right| \leq C$ on $\partial B_{\delta_{0}}\left(p_{k, j}\right)$. This implies $\max _{x \in B_{\delta_{0}}}\left(p_{k, j}\right) u_{k}(x) \leq C$, which contradicts to our assumption.

Next, we present a theorem which gives (P3)-sup + inf estimates. The proof of it is interesting and given in a separate section.

Lemma 2.4. We have

$$
\left|u_{k}(x)-u_{k}\left(p_{k, j}\right)-\log \frac{\alpha_{4}}{\left(1+\frac{\left|x-p_{k, j}\right|^{2}}{\epsilon_{k, j}^{2}}\right)^{4}}\right| \leq C,
$$

for $x \in B_{\delta_{0}}\left(p_{k, j}\right)$.

From Lemma 2.4, we have the following important corollary:

Corollary 2.5. Let $u_{k}$ be a sequence of blowup solutions of (1.1) with $\rho=\rho_{k}$. Let $l_{k}, l_{k, j}, \epsilon_{k}, \epsilon_{k, j}$ be defined as before. It then holds

$$
\begin{aligned}
& l_{k}-C \leq l_{k, j} \leq l_{k}+C, C^{-1} \epsilon_{k} \leq \epsilon_{k, j} \leq C \epsilon_{k}, j=1, \ldots, m, \\
& c_{k}-C \leq l_{k, j} \leq c_{k}+C, C^{-1} e^{-\frac{c_{k}}{4}} \leq \epsilon_{k, j} \leq C e^{-\frac{c_{k}}{4}}, j=1, \ldots, m .
\end{aligned}
$$

Finally, we consider a problem in $\mathbb{R}^{4}$. It has been proved $[15,22]$ that the solution to the following problem

$$
\left\{\begin{array}{l}
\Delta^{2} U=e^{U}, \quad \text { in } \mathbb{R}^{4}, \\
\int_{\mathbb{R}^{4}} e^{U}<+\infty,
\end{array}\right.
$$

is given by

$$
U_{\epsilon, a}(x):=\log \frac{\alpha_{4} \epsilon^{4}}{\left(\epsilon^{2}+|x-a|^{2}\right)^{4}},
$$

for any $\epsilon>0, a \in \mathbb{R}^{4}$, provided that

$$
U(x)=o\left(|x|^{2}\right) \text { as }|x| \rightarrow+\infty .
$$

Let $U=\log \frac{\alpha_{4}}{\left(1+|y|^{2}\right)^{4}}$ and $\tau \in(0,1)$ be a fixed constant. We need the following lemma which proves the nondegeneracy of $U$ : 
Lemma 2.6. The solutions to the following linearized problem

$$
\Delta^{2} \phi=e^{U} \phi, \quad|\phi(y)| \leq C(1+|y|)^{\tau}
$$

is given by $\phi=\sum_{j=0}^{4} c_{j} \psi_{j}$ where

$$
\psi_{0}=\frac{1-|y|^{2}}{1+|y|^{2}}, \psi_{j}=\frac{y_{j}}{1+|y|^{2}}, j=1, \ldots, 4 .
$$

\section{Proof of Lemma 2.4}

In this section, we prove the sup+inf estimates-Lemma 2.4. As we mentioned before, the method of moving spheres seems not applicable here. Instead, we use an approach of combination of potential analysis and Pohozaev identity. This approach has been used in Bartolucci-Chen-Lin-Tarantello [4].

We now state a more general theorem: Let $\tilde{u}_{k}(x)$ be a solution of

$$
\left\{\begin{array}{l}
\triangle^{2} \tilde{u}_{k}(x)=h_{k}(x) e^{\tilde{u}_{k}} \text { in } B_{2}, \text { and } \\
\int_{B_{2}} h_{k}(x)^{e^{\tilde{u}_{k}(x)}} d x \leq C,
\end{array}\right.
$$

where $h_{k}(x)$ converges to a positive function $h(x)$ in $C^{1}\left(\bar{B}_{2}\right)$, and without loss of generality, we may assume $h(0)=1$. Suppose that $\tilde{u}_{k}$ satisfies the following assumptions

(i) $\left|\tilde{u}_{k}(x)-\tilde{u}_{k}(y)\right| \leq c$ for $|x|=|y|=2$,

(ii) $\left|\triangle \tilde{u}_{k}(x)\right|$ is bounded in any compact set of $\bar{B}_{2} \backslash\{0\}$,

(iii) 0 is the only blow-up point of $\tilde{u}_{k}$, i.e., set $S=\left\{x \mid x_{k} \rightarrow x\right.$ and $\overline{\lim }_{k \rightarrow+\infty} \tilde{u}_{k}\left(x_{k}\right) \rightarrow$ $+\infty\}$. Then $S=\{0\}$.

We want to establish the following sharp estimate of the bubbling behavior of $\tilde{u}_{k}$ near 0 . To state our result, we let $l_{k}$ be the maximum and $x_{k}$ be a maximum point of $\tilde{u}_{k}$, i.e.,

$$
l_{k}=\tilde{u}_{k}\left(x_{k}\right)=\max _{\bar{B}_{2}} \tilde{u}_{k} .
$$

and let $v(x)$ be the solution of

$$
\begin{aligned}
& \triangle^{2} v(x)=e^{v(x)} \text { in } \mathbb{R}^{4} \\
& v(0)=0=\max _{\mathbb{R}^{2}} v(x) \text { and }|v(x)|=O(\log |x|) \text { at } \infty .
\end{aligned}
$$

Theorem 3.1. Suppose $\tilde{u}_{k}$ is a sequence of solution of (3.1) and satisfies assumptions (i)-(iii) and $v$ is the solution of (3.2). Then there exists a constant $c$ such that

$$
\left|\tilde{u}_{k}(x)-l_{k}-v\left(e^{\frac{l_{k}}{4}}\left|x-x_{k}\right|\right)\right| \leq C \text { in } \bar{B}_{1} .
$$


Applying Theorem 3.1 to $\tilde{u}_{k}=u_{k}-c_{k}$, we obtain Lemma 2.4.

For $r \in(0,1)$, set

$$
\alpha_{k}(r)=\int_{B_{r}} h_{k}(x) e^{\tilde{u}_{k}(x)} d x
$$

and

$$
\alpha(r)=\lim _{k \rightarrow+\infty} \alpha_{k}(r) \text { and } \alpha=\lim _{r \rightarrow 0} \alpha(r)
$$

We first have:

Lemma 3.2. Suppose $\tilde{u}_{k}$ is a solution of (3.1) and satisfies assumption (i)-(iii). Then $\tilde{u}_{k} \rightarrow-\infty$ uniformly in any compact set and $\alpha=32 \sigma_{3}$.

Proof. Suppose that there exists a point $x_{0} \in B_{2} \backslash\{0\}$ such that $\tilde{u}_{k}\left(x_{0}\right)$ is bounded. Then by assumptions (i)-(iii), the sequence $\tilde{u}_{k}$ is bounded in any compact set of $B_{2} \backslash$ $\{0\}$. By taking a diagonal process, a subsequence, still denoted by $\tilde{u}_{k}$, approaches a function $u(x)$ in $B_{2} \backslash\{0\}$ which satisfies

$$
\triangle^{2} u(x)=h(x) e^{u(x)} \text { in } B_{2} \backslash\{0\} .
$$

On the other hand, fixing any $\delta>0$ and small, we integrate (3.1) in $B_{\delta}$ and obtain

$$
\int_{\partial B_{\delta}} \frac{\partial \Delta \tilde{u}_{k}}{\partial v}=\int_{B_{\delta}} \Delta^{2} \tilde{u}_{k}=\int_{B_{\delta}} h_{k} e^{\tilde{u}_{k}(x)} d x=\alpha_{k}(\delta)
$$

which implies that

$$
\lim _{\delta \rightarrow 0} \int_{\partial B_{\delta}} \frac{\partial \Delta u}{\partial v}=\alpha .
$$

Therefore $u(x)$ satisfies (in the distribution sense)

$$
\triangle^{2} u(x)=h(x) e^{u(x)}+\alpha \delta_{0} \text { in } B_{2} .
$$

Thus

$$
\begin{aligned}
& u(x)=\frac{\alpha}{4 \sigma_{3}} \log \left(\frac{1}{|x|}\right)+v(x), \text { with } \alpha>0, v(x) \text { is smooth, and } \\
& * \int_{B_{1}} e^{u(x)} d x \leq C .
\end{aligned}
$$

By the Pohozaev identity (2.5), we have

$$
\begin{aligned}
& \int_{B_{r}}\left[4 h_{k}(x)+\left(\nabla h_{k}(x) \cdot x\right)\right] e^{\tilde{u}_{k}} \\
= & \int_{\partial B_{r}} h_{k}(x)|x| e^{\tilde{u}_{k}} d \sigma-\int_{\partial B_{r}} r\left[\frac{\left(\triangle \tilde{u}_{k}\right)^{2}}{2}+\frac{\partial \tilde{u}_{k}}{\partial r} \frac{\partial}{\partial r} \Delta \tilde{u}_{k}\right] d \sigma \\
& +\int_{\partial B_{r}} \frac{\partial}{\partial r}\left(r \frac{\partial \tilde{u}_{k}}{\partial r}\right) \triangle \tilde{u}_{k} d \sigma .
\end{aligned}
$$


By letting $k \rightarrow+\infty$, we have

$$
4 \alpha(1+o(1))=2\left(\frac{\alpha}{4 \sigma_{3}}\right)^{2} \sigma_{3}(1+o(1)),
$$

where $o(1)$ tends to 0 as $r \rightarrow 0$. Since $\alpha>0$, we have

$$
\alpha=32 \sigma_{3}
$$

However, (3.8) implies $\frac{\alpha}{4 \sigma_{3}}=8$ and then (3.1) yields

$$
\int_{B_{1}}|x|^{-8} d x \leq c_{1} \int_{B_{1}} e^{u(x)} d x \leq c_{1} c
$$

a contradiction. Thus, $\tilde{u}_{k}(x) \rightarrow-\infty$ uniformly in any compact set of $B_{2} \backslash\{0\}$.

Now it is obvious that $\hat{u}_{k}(x)=\tilde{u}_{k}(x)-c_{k}$ converges to $\hat{u}(x)$ in $C_{l o c}^{2}\left(\bar{B}_{2} \backslash\{0\}\right)$ and $c_{k} \rightarrow+\infty$ as $k \rightarrow+\infty$ where $c_{k}=f_{|x|=1} \tilde{u}_{k}(x) d \sigma$ is the average of $\tilde{u}_{k}$ over $S^{3}$. Clearly

$$
\hat{u}(x)=\frac{\alpha}{4 \sigma_{3}} \log \frac{1}{|x|}+v(x),
$$

with $\triangle^{2} v(x)=0$ in $B_{2}$. We can apply the Pohozaev identity (3.6) to obtain $\alpha=$ $32 \sigma_{3}$ as the same as (3.8). Thus, Lemma 3.2 is proved.

Proof of Theorem 3.1. By (i) and (ii), it is easy to see that $\tilde{u}_{k}(x)$ can be written as

$$
\tilde{u}_{k}(x)=\frac{1}{4 \sigma_{3}} \int_{B_{2}} \log \left(\frac{1}{|x-y|}\right) h_{k}(y) e^{\tilde{u}_{k}(y)} d y+f_{k}(x),
$$

where $f_{k}(x)$ is a smooth function in $\bar{B}_{\frac{3}{2}}$, and

$$
\left\|f_{k}\right\|_{C^{4}\left(\bar{B}_{\frac{3}{2}}\right)} \leq C .
$$

Recall $\tilde{u}_{k}\left(x_{k}\right)=l_{k}=\max _{\bar{B}_{2}} \tilde{u}_{k}$. Then

$$
\tilde{u}_{k}(x)-l_{k}=\frac{1}{4 \sigma_{3}} \int_{B_{2}} \log \left\{\frac{\left|x_{k}-y\right|}{|x-y|}\right) h_{k}(y) e^{\tilde{u}_{k}(y)} d y+f_{k}(x)-f_{k}\left(x_{k}\right) .
$$

Set $v_{k}(x)=\tilde{u}_{k}\left(\varepsilon_{k} x+x_{k}\right)-l_{k}$ and $\varepsilon_{k}=e^{-\frac{l_{k}}{4}}$. Then (3.12) implies

$$
v_{k}(x)=\frac{1}{4 \sigma_{3}} \int_{B_{\varepsilon_{k}-1}} \log \left(\frac{|y|}{|x-y|}\right) \tilde{h}_{k}(y) e^{v_{k}(y)} d y+\tilde{f}_{k}(x),
$$


where $\tilde{h}_{k}(y)=h_{k}\left(x_{k}+e^{-\frac{l_{k}}{4}} y\right)$ and

$$
\left\|\nabla^{j} \tilde{f}_{k}\right\|_{B_{\varepsilon_{k}}^{-1}} \rightarrow 0 \text { for } 1 \leq j \leq 4 .
$$

From (3.13), we have $\left|\Delta v_{k}(x)\right|$ is uniformly bounded. Thus, $v_{k}(x) \rightarrow v(x)$ in $C_{\text {loc }}^{4}\left(\mathbb{R}^{4}\right)$ and $v(x)$ satisfies

$$
\begin{aligned}
& \triangle^{2} v(x)=e^{v(x)} \text { in } \mathbb{R}^{4} \text { and } \\
& v(x)=\frac{1}{4 \sigma_{3}} \int_{\mathbb{R}^{4}} \log \left(\frac{|y|}{|x-y|}\right) e^{v(y)} d y+c_{0}
\end{aligned}
$$

for some constant $c_{0}$. Therefore $\Delta v(x) \rightarrow 0$ as $|x| \rightarrow+\infty$, a classification result of [2] shows that

$$
v(x)=c+\log \frac{1}{\left(1+|x|^{2}\right)^{4}} .
$$

Thus, for any $R>0$,

$$
\left|v_{k}(x)-v(x)\right| \rightarrow 0 \text { uniformly for }|x| \leq R
$$

as $k \rightarrow+\infty$.

To prove Theorem 3.1, it is equivalent to showing

$$
\left|v_{k}(x)-v(x)\right| \leq C \text { for } R \leq|x| \leq r_{0} e^{\frac{l_{k}}{4}},
$$

for some $r_{0}>0$.

To prove (3.16), we claim

$$
\left|\alpha_{k}-32 \sigma_{3}\right| \leq c\left(\log \frac{1}{\varepsilon_{k}}\right)^{-1}
$$

where $\alpha_{k}$ is the local mass defined by

$$
\alpha_{k}=\int_{B_{1}} h_{k}(x) e^{\tilde{u}_{k}(x)} d x
$$

The idea to obtain (3.17) is to apply the Pohozaev identity (3.6) on the circle $|x|=$ $\varepsilon_{k}\left(\log \frac{1}{\varepsilon_{k}}\right)$. Hence, we need some fine estimates of $v_{k}$. Basically, all estimates required here can be obtained by using the Green representation formulas (3.13). First, we has a rough estimate about the behavior of $v_{k}$.

For any fixed $\delta>0$, there exists $R=R_{\delta}$ and $k_{0}=k(\delta) \in N$ such that if $|x| \geq 2 R$ and $k \geq k_{0}$, then

$$
v_{k}(x) \leq-\left(\frac{\alpha_{k}}{4 \sigma_{3}}-\delta\right) \log |x|
$$


The proof is standard, and is omitted here. Since $\frac{\alpha_{k}}{4 \sigma_{3}} \rightarrow 8$ as $k \rightarrow+\infty, \delta$ is always chosen such that

$$
v_{k}(x) \leq-7 \log |x| \text { holds for }|x| \geq 2 R .
$$

For $\log \frac{1}{\varepsilon_{k}} \leq|x| \leq \varepsilon_{k}^{-1}$, set

$$
\tilde{\alpha}_{k}(|x|)=\int_{|y| \leq r_{o}|x|} \tilde{h}_{k} e^{v_{k}(y)} d y,
$$

where $r_{0} \leq \frac{1}{2}$ is a positive constant. By (3.19),

$$
\left|\alpha_{k}-\tilde{\alpha}_{k}(|x|)\right| \leq c \int_{|y| \geq r_{0}|x|} e^{v_{k}(y)} d y \leq c \int_{|y| \geq r_{o}|x|}|y|^{-7} d y=O\left(\frac{1}{|x|^{3}}\right),
$$

for $|x| \geq \log \frac{1}{\varepsilon_{k}}$ and $k$ large.

By (3.20), we claim that

$$
\begin{aligned}
\left|v_{k}(x)+\frac{\alpha_{k}}{4 \sigma_{3}} \log \right| x|| & \leq C, \\
\left|\frac{\partial v_{k}}{\partial r}(x)+\frac{\alpha_{k}}{4 \sigma_{3}} \frac{1}{|x|}\right| & \leq O\left(\left(\log \frac{1}{\varepsilon_{k}}\right)^{-1}|x|^{-1}\right) \\
\left|\frac{\partial}{\partial r}\left(r \frac{\partial v_{k}}{\partial r}(x)\right)\right| & \leq O\left(\frac{1}{|x|^{2}}\right) \\
\left|\triangle v_{k}(x)+\frac{\alpha_{k}}{2 \sigma_{3}} \frac{1}{|x|^{2}}\right| & \leq O\left(\left(\log \frac{1}{\varepsilon_{k}}\right)^{-1}|x|^{-2}\right), \text { and } \\
\left|\frac{\partial}{\partial r} \Delta v_{k}(x)-\frac{\alpha_{k}}{\sigma_{3}} \frac{1}{|x|^{3}}\right| & \leq C\left(\log \frac{1}{\varepsilon_{k}}\right)^{-1}|x|^{-3},
\end{aligned}
$$

for $|x|=\log \frac{1}{\varepsilon_{k}}$. In fact, we will prove (3.21) holds for $\log \frac{1}{\varepsilon_{k}} \leq|x| \leq \frac{1}{\varepsilon_{k}}$.

We first show (3.17) by assuming (3.21)-(3.25). Rescaling back to $\tilde{u}_{k},(3.21)$ (3.25) can be written as follows:

$$
\tilde{u}_{k}(x)=v_{k}\left(\varepsilon_{k}^{-1} x\right)-4 \log \varepsilon_{k}=-\frac{\alpha_{k}}{4 \sigma_{3}} \log |x|+\left(\frac{\alpha_{k}}{4 \sigma_{3}}-4\right) \log \varepsilon_{k}
$$




$$
\begin{aligned}
\frac{\partial \tilde{u}_{k}(x)}{\partial r} & =-\frac{\alpha_{k}}{4 \sigma_{3}} \frac{1}{|x|}+O\left(\left(\log \frac{1}{\varepsilon_{k}}\right)^{-1} \frac{1}{|x|}\right), \\
\left|\frac{\partial}{\partial r}\left(r \frac{\partial \tilde{u}_{k}}{\partial r}(x)\right)\right| & \leq O\left(\left(\log \frac{1}{\varepsilon_{k}}\right)^{-1} \frac{1}{|x|}\right) \\
\triangle \tilde{u}_{k}(x) & =-\frac{\alpha_{k}}{2 \sigma_{3}} \frac{1}{|x|^{2}}+O\left(\left(\log \frac{1}{\varepsilon_{k}}\right)^{-1} \frac{1}{|x|^{2}}\right), \text { and } \\
\frac{\partial}{\partial r} \triangle \tilde{u}_{k}(x) & =\frac{\alpha_{k}}{\sigma_{3}} \frac{1}{|x|^{3}}+O\left(\left(\log \frac{1}{\varepsilon_{k}}\right)^{-1} \frac{1}{|x|^{3}}\right)
\end{aligned}
$$

for $|x|=2 k\left(\log \frac{1}{\varepsilon_{k}}\right)$.

Substituting (3.26)-(3.30) to (3.6) on $r=\varepsilon_{k} \log \left(\frac{1}{\varepsilon_{k}}\right)$, we have

$$
4 \alpha_{k}+O(1) \varepsilon_{k} \log \left(\frac{1}{\varepsilon_{k}}\right)=\frac{\alpha_{k}^{2}}{8 \sigma_{3}}+O\left(\left(\log \frac{1}{\varepsilon_{k}}\right)^{-1}\right) \text {. }
$$

Thus,

$$
\alpha_{k}=32 \sigma_{3}+O\left(\left(\log \frac{1}{\varepsilon_{k}}\right)^{-1}\right),
$$

and then (3.17) is proved.

We come back for the proof of (3.21)-(3.25). By (3.13), for $\log \varepsilon_{k}^{-1} \leq|x| \leq \varepsilon_{k}^{-1}$,

$$
\begin{aligned}
v_{k}(x) & =\frac{1}{4 \sigma_{3}} \int_{|y| \leq \varepsilon_{k}^{-1}} \log \left(\frac{|y|}{|x-y|}\right) \tilde{h}_{k}(y) e^{v_{k}(y)} d y+O(1) \\
& =\frac{1}{4 \sigma_{3}} \int_{|y| \leq r_{0}|x|} \log \left(\frac{1}{|x-y|}\right) \tilde{h}_{k}(y) e^{v_{k}(y)} d y+O(1) \\
& =\frac{\tilde{\alpha}_{k}}{4 \sigma_{3}} \log \frac{1}{|x|}+O(1) \\
& =\frac{\alpha_{k}}{4 \sigma_{3}} \log \frac{1}{|x|}+O(1)
\end{aligned}
$$

where

$$
\begin{aligned}
& \int_{|y| \leq e_{k}^{-1}}|\log (|y|)| \tilde{h}_{k}(y) e^{v_{k}(y)} d y \leq c, \\
& \int_{|y| \geq r_{0}|x|} \log \left(\frac{1}{|x-y|}\right) \tilde{h}_{k}(y) e^{v_{k}(y)} d y=O\left(|x|^{-3} \log |x|\right),
\end{aligned}
$$


and (3.20) are employed. This proves (3.21). To prove (3.22), we have

$$
\begin{aligned}
\frac{\partial v_{k}(x)}{\partial r}= & \frac{-1}{4 \sigma_{3}} \int_{|y| \leq \varepsilon_{k}^{-1}} \frac{(x-y) \cdot \frac{x}{|x|}}{|x-y|^{2}} \tilde{h}_{k}(y) e^{v_{k}(y)} d y+O\left(\varepsilon_{k}\right) \\
= & \frac{-1}{4 \sigma_{3}} \int_{|y| \leq r_{0}|x|} \frac{(x-y) x}{|x||x-y|^{2}} \tilde{h}_{k}(y) e^{v_{k}(y)} d y+O\left(|x|^{-4}+\varepsilon_{k}\right) \\
= & \frac{-1}{4 \sigma_{3}} \int_{|y| \leq r_{0}|x|} \frac{1}{|x|} \tilde{h}_{k}(y) e^{v_{k}(y)} d y+\frac{O(1)}{|x|^{2}} \int_{|y| \leq r_{0}|x|}|y| e^{v_{k}(y)} d y \\
& +O\left(|x|^{-4}+\varepsilon_{k}\right) \\
= & -\frac{\tilde{\alpha}_{k}}{4 \sigma_{3}} \frac{1}{|x|}+O\left(|x|^{-2}\right) \\
= & -\frac{\alpha_{k}}{4 \sigma_{3}}|x|^{-1}+O\left(\left(\log \frac{1}{\varepsilon_{k}}\right)^{-1}|x|^{-1}\right) .
\end{aligned}
$$

For (3.23), we have

$$
\begin{aligned}
\frac{\partial}{\partial r}\left(r \frac{\partial v_{k}}{\partial r}(r)\right) & =\frac{-1}{4 \sigma_{3}} \int_{|y| \leq \varepsilon_{k}^{-1}} \frac{\partial}{\partial r}\left(\frac{(x-y) x}{|x-y|^{2}}\right) \tilde{h}_{k}(y) e^{v_{k}(y)} d y+O\left(\varepsilon_{k}\right) \\
& =\frac{-1}{4 \sigma_{3}} \int_{|y| \leq r_{0}|x|} \frac{\partial}{\partial r}\left(\frac{(x-y) x}{|x-y|^{2}}\right) \tilde{h}_{k}(y) e^{v_{k}(y)} d y+O\left(\varepsilon_{k}+|x|^{-4}\right) \\
& =\frac{O(1)}{|x|^{2}} \int_{|y| \leq r_{0}|x|}|y|(1+|y|)^{-6} d y+O\left(\varepsilon_{k}+|x|^{-4}\right) \\
& =O\left(\frac{1}{|x|^{2}}\right) .
\end{aligned}
$$

We have proved (3.21)-(3.23). Proofs of (3.24) and (3.25) are similar, and we should omit them here. Hence (3.17) is proved completely.

We note (3.21) holds for $\log \frac{1}{\varepsilon_{k}} \leq|x| \leq \varepsilon_{k}^{-1}$. Therefore, by (3.17) and (3.21), we have

$$
\left|v_{k}(x)+8 \log \right| x|| \leq C
$$


for $\log \frac{1}{\varepsilon_{k}} \leq|x| \leq \varepsilon_{k}^{-1}$. So far, we have proved

$$
\left|v_{k}(x)-v(x)\right| \leq C \text { for }|x| \leq R \text { or }|x| \geq \log \frac{1}{\epsilon_{k}} .
$$

For the region $R \leq|x| \leq \frac{1}{\varepsilon_{k}}$, we proceeds as follows: for $|x| \leq \log \frac{1}{\epsilon_{k}}$,

$$
\begin{aligned}
\Delta v_{k}(x)= & -\frac{1}{2 \sigma_{3}} \int_{|y| \leq r_{o}|x|} \frac{1}{|x-y|^{2}} \tilde{h}_{k}(y) e^{v_{k}(y)} d y+O\left(|x|^{-5}\right) \\
= & -\frac{1}{2 \sigma_{3}} \int_{|y| \leq r_{o}|x|} \frac{1}{|x|^{2}} \tilde{h}_{k}(y) e^{v_{k}(y)} d y \\
& -\frac{1}{\sigma_{3}} \int_{|y| \leq r_{o}|x|} \frac{x \cdot y}{|x|^{4}} \tilde{h}_{k}(y) e^{v_{k}(y)} d y+O\left(\frac{1}{|x|^{4}}\right) \\
= & -\frac{\tilde{\alpha}_{k}}{2 \sigma_{3}} \frac{1}{|x|^{2}}+O\left(\frac{1}{|x|^{3}}\right) \\
= & \Delta v(x)+O\left(\frac{1}{|x|^{3}}\right)
\end{aligned}
$$

where we have used the fact that $\tilde{\alpha}_{k}(|x|)-\alpha_{k}=O\left(\frac{1}{|x|^{3}}\right)$ and that $\alpha_{k}-32 \sigma_{3}=$ $O\left(\frac{1}{\log \frac{1}{\epsilon_{k}}}\right)$.

Thus,

$$
\left|\Delta v_{k}(x)-\Delta v(x)\right| \leq \frac{C}{|x|^{3}}
$$

holds for $R \leq|x| \leq \frac{1}{\epsilon_{k}}$.

Now we choose $g(x)=C\left(1+\frac{1}{|x|}\right)$ where $C$ is large. Then $-\Delta g(x)=\frac{C}{|x|^{3}} \geq$ $\left|\Delta v_{k}(x)-\Delta v(x)\right|$. It is easy to see that $g(x) \geq\left|v_{k}(x)-v(x)\right|$ for $|x|=R$ and $|x|=\log \frac{1}{\epsilon_{k}}$. Thus, the maximum principle implies

$$
\left|v_{k}(x)-v(x)\right| \leq C\left(1+\frac{1}{|x|}\right)
$$

for $R \leq|x| \leq \log \frac{1}{\epsilon_{k}}$.

Combining (3.31) and (3.33), we have proved (3.16). Thus, Theorem 3.1 is completely proved. 


\section{The Estimate of $\rho_{k}-32 \sigma_{4} m$}

The main purpose of this section is to prove Theorem 1.2. We follow the main steps used in [7]. Let

$$
\tilde{u}_{k}(x)=u_{k}(x)-c_{k}
$$

which satisfies

$$
\Delta^{2} \tilde{u}_{k}=\rho_{k} h(x) e^{\tilde{u}_{k}} \text { in } \Omega, \quad \int_{\Omega} h(x) e^{\tilde{u}_{k}}=1 .
$$

Recall the definitions $c_{k}, p_{k, j}, l_{k}, l_{k, j}, \epsilon_{k}, \epsilon_{k, j}$ given in (2.7)-(2.9).

For a fixed small $\delta_{0}>0$, we set the local "mass" $\rho_{k, j}$ to be

$$
\rho_{k, j}=\rho_{k} \int_{B_{\delta_{0}}\left(p_{j}\right)} h(x) e^{\tilde{u}_{k}(x)} d x
$$

By Corollary 2.5, we have

$$
\rho_{k, j}=\rho_{k} \int_{B_{\delta_{0}}\left(p_{k, j}\right)} h(x) e^{\tilde{u}_{k}(x)} d x+O\left(\epsilon_{k}^{4}\right)
$$

which yields

$$
\sum_{j=1}^{m} \rho_{k, j}=\rho_{k}+O\left(\epsilon_{k}^{4}\right)
$$

In $B_{\delta_{0}}\left(p_{k, j}\right)$, we set

$$
G_{j}^{*}(x)=\rho_{k, j} R_{4}\left(x, p_{k, j}\right)+\sum_{l \neq j} \rho_{k, l} G_{4}\left(x, p_{k, l}\right)
$$

and $w_{k}(x)$ to be the error term defined by

$$
w_{k}(x)=u_{k}(x)-\sum_{i=1}^{m} \rho_{k, i} G_{4}\left(x, p_{k, i}\right)=\tilde{u}_{k}-\sum_{i=1}^{m} \rho_{k, i} G_{4}\left(x, p_{k, i}\right)+c_{k}
$$

on $\Omega \backslash \cup_{j=1}^{m} B_{\frac{\delta_{0}}{2}}\left(p_{j}\right)$.

We first have:

Estimate A. $\left|w_{k}(x)\right|+\left|\partial^{\alpha} w_{k}(x)\right|=O\left(\epsilon_{k}\right)$ for all $|\alpha| \leq 3, x \in \Omega \backslash \cup_{j=1}^{m} B_{\frac{\delta_{0}}{2}}\left(p_{j}\right)$. 
Proof. This follows from the Green's representation formula:

$$
\begin{aligned}
& u_{k}(x)= \int_{\Omega} G_{4}(x, y)\left(\rho_{k} h(y) e^{\tilde{u}_{k}}\right) d y \\
&= \sum_{j=1}^{m} \rho_{k} \int_{B_{\frac{\delta_{0}}{2}}\left(p_{k, j}\right)} G_{4}(x, y) h(y) e^{\tilde{u}_{k}}+O\left(\epsilon_{k}^{4}\right) \\
&= \sum_{j=1}^{m} \rho_{k} \int_{B_{\frac{\delta_{0}}{2}}\left(p_{k, j}\right)}\left[G_{4}(x, y)-G_{4}\left(x, p_{k, j}\right)\right] h(y) e^{\tilde{u}_{k}} \\
&+\sum_{j=1}^{m} \rho_{k} G_{4}\left(x, p_{k, j}\right) \int_{B_{\frac{\delta_{0}}{2}}}\left(p_{k, j}\right) \\
& h(y) e^{\tilde{u}_{k}}+O\left(\epsilon_{k}^{4}\right) \\
&= \sum_{j=1}^{m} \rho_{k} \int_{B_{\frac{\delta_{0}}{2}}\left(p_{k, j}\right)} O\left(\left|y-p_{k, j}\right|\right) h(y) e^{\tilde{u}_{k}}+\sum_{j=1}^{m} \rho_{k, j} G_{4}\left(x, p_{k, j}\right)+O\left(\epsilon_{k}^{4}\right) \\
&= \sum_{j=1}^{m} \rho_{k, j} G_{4}\left(x, p_{k, j}\right)+O\left(\epsilon_{k}\right) .
\end{aligned}
$$

Similarly we can estimate $\left|\partial^{\alpha} w_{k}(x)\right|$ for $|\alpha| \leq 3$.

Hence Estimate A is established.

Estimate B. $\left|\nabla\left(\log h(x)+G_{j}^{*}(x)\right)\right|=O\left(\epsilon_{k}\right)$ at $x=p_{k, j}$.

Proof. Applying (2.6) to $\tilde{u}_{k}$ on $B_{\delta_{0}}\left(p_{k, j}\right)$, we obtain

$$
\begin{aligned}
\operatorname{LHS} \text { of }(2.6)= & \rho_{k} \int_{B_{\delta_{0}}\left(p_{k, j}\right)}<\xi, \nabla h>e^{\tilde{u}_{k}} \\
= & \rho_{k} \int_{B_{\delta_{0}}\left(p_{k, j}\right)}\left[<\xi, \nabla h>-<\xi, \nabla h\left(p_{k, j}\right)>\right] e^{\tilde{u}_{k}} \\
& +\rho_{k} \int_{B_{\delta_{0}}\left(p_{k, j}\right)}<\xi, \nabla h\left(p_{k, j}\right)>e^{\tilde{u}_{k}} \\
= & O\left(\epsilon_{k}\right)+\rho_{k} \int_{B_{\delta_{0}}\left(p_{k, j}\right)} h\left(p_{k, j}\right) e^{\tilde{u}_{k}}<\xi, \nabla \log h\left(p_{k, j}\right)> \\
= & O\left(\epsilon_{k}\right)+\rho_{k, j}<\xi, \nabla \log h\left(p_{k, j}\right)>.
\end{aligned}
$$


On the other hand by Estimate A, we have

$$
u_{k}(x)=w_{k}(x)+\hat{G}_{j}^{*}(x)
$$

where

$$
\hat{G}_{j}^{*}(x)=G_{j}^{*}(x)+\frac{\rho_{k, j}}{4 \sigma_{3}} \log \frac{1}{\left|x-p_{k, j}\right|} .
$$

Note that $\Delta^{2}\left(\hat{G}_{j}^{*}(x)\right)=0$ in $B_{\delta_{0}}\left(p_{k, j}\right) \backslash\left\{p_{k, j}\right\}$. Applying the Pohozaev's identity to $\hat{G}_{j}^{*}$, we obtain

RHS of (2.6)

$$
\begin{aligned}
= & \int_{\partial B_{\delta_{0}}\left(p_{j}\right)}\left[<\xi, v>\left(\frac{1}{2}\left|\Delta u_{k}\right|^{2}\right)-<\xi, \nabla \Delta u_{k}>\frac{\partial u_{k}}{\partial r}-<\xi, \nabla u_{k}>\frac{\partial \Delta u_{k}}{\partial r}\right. \\
& \left.+<\xi, v>\frac{\partial u_{k}}{\partial r} \frac{\partial \Delta u_{k}}{\partial r}\right]+O\left(\epsilon_{k}^{4}\right) \\
= & \int_{\partial B_{\delta_{0}}\left(p_{j}\right)}\left[<\xi, v>\left(\frac{1}{2}\left|\Delta \hat{G}_{j}^{*}\right|^{2}\right)-<\xi, \nabla \Delta \hat{G}_{j}^{*}>\frac{\partial \hat{G}_{j}^{*}}{\partial r}<\xi, \nabla \hat{G}_{j}^{*}>\frac{\partial \Delta \hat{G}_{j}^{*}}{\partial r}\right. \\
& \left.+<\xi, v>\frac{\partial \hat{G}_{j}^{*}}{\partial r} \frac{\partial \Delta \hat{G}_{j}^{*}}{\partial r}\right]+O\left(\epsilon_{k}\right) \\
= & \lim _{r \rightarrow 0} \int_{\partial B_{r}\left(p_{j}\right)}\left[<\xi, \nu>\left(\frac{1}{2}\left|\Delta \hat{G}_{j}^{*}\right|^{2}\right)-<\xi, \nabla \Delta \hat{G}_{j}^{*}>\frac{\partial \hat{G}_{j}^{*}}{\partial r}-<\xi, \nabla \hat{G}_{j}^{*}>\frac{\partial \Delta \hat{G}_{j}^{*}}{\partial r}\right. \\
& \left.+<\xi, v>\frac{\partial \hat{G}_{j}^{*}}{\partial r} \frac{\partial \Delta \hat{G}_{j}^{*}}{\partial r}\right]+O\left(\epsilon_{k}\right) \\
= & -\rho_{k, j}<\xi, \nabla G_{j}^{*}\left(p_{k, j}\right)>+O\left(\epsilon_{k}\right) .
\end{aligned}
$$

This proves Estimate B.

Next, we give a sharper description of the bubbling behavior of $u_{k}$ in the ball $B_{\delta_{0}}\left(p_{k, j}\right)$. We set

$$
h_{k, j}=h\left(p_{k, j}\right)
$$

and

$$
v_{k, j}(x)=\log \left(\frac{\alpha_{4} \epsilon_{k, j}^{4}}{\left(\epsilon_{k, j}^{2}+\sqrt{\rho_{k} h_{k, j}}\left|x-q_{k, j}\right|^{2}\right)^{4}}\right)
$$


where $\epsilon_{k, j}$ is given by (2.8) and $q_{k, j} \in \mathbb{R}^{4}$ is chosen such that

$$
\nabla v_{k, j}\left(p_{k, j}\right)=\nabla \log h\left(p_{k, j}\right)
$$

By direct computations, we also have

$$
\left|p_{k, j}-q_{k, j}\right|=O\left(\epsilon_{k}^{2}\right)
$$

For $x \in B_{\delta_{0}}\left(p_{k, j}\right)$, we also set

$$
\eta_{k, j}(x)=u_{k}(x)-c_{k}-v_{k, j}(x)-\left(G_{j}^{*}(x)-G_{j}^{*}\left(p_{k, j}\right)\right) .
$$

Then by Estimate B and (4.11), we have

$$
\begin{aligned}
\left|\nabla \eta_{k, j}\left(p_{k, j}\right)\right| & =O\left(\epsilon_{k}\right) \\
\eta_{k, j}\left(p_{k, j}\right) & =l_{k, j}-v_{k, j}\left(p_{k, j}\right)=O\left(\epsilon_{k}\right) \\
\mid \eta_{k, j}(x) & \leq C \text { for } x \in B_{\delta_{0}}\left(p_{k, j}\right) .
\end{aligned}
$$

Let us check the matching of $w_{k}$ and $\eta_{k, j}$ on $B_{\frac{\delta}{2}}\left(p_{k, j}\right)$ :

$$
\begin{aligned}
& \eta_{k, j}(x)=u_{k}(x)-c_{k}-v_{k, j}(x)-\left(G_{j}^{*}(x)-G_{j}^{*}\left(p_{k, j}\right)\right) \\
& =w_{k}+\sum_{i} \rho_{k, i} G_{4}\left(x, p_{k, i}\right)-c_{k} \\
& -\log \frac{\alpha_{4} \epsilon_{j}^{4}}{\left(\epsilon_{j}^{2}+\sqrt{\rho_{k} h_{k, j}}\left|x-q_{k, j}\right|^{2}\right)^{4}}-\left(G_{j}^{*}(x)-G_{j}^{*}\left(p_{k, j}\right)\right) \\
& =O\left(\epsilon_{k}\right)+\rho_{k, j} G_{4}\left(x, p_{k, j}\right)+\rho_{k, j} R_{4}\left(x, p_{k, j}\right)-c_{k}+G_{j}^{*}\left(p_{k, j}\right) \\
& -\log \frac{\alpha_{4} \epsilon_{k, j}^{4}}{\left(\sqrt{\rho_{k} h_{k, j}}\left|x-q_{k, j}\right|^{2}\right)^{4}} \\
& =O\left(\epsilon_{k}\right)+\left(\frac{\rho_{k, j}}{4 \sigma_{3}}-8\right) \log \frac{1}{\left|x-p_{k, j}\right|}-c_{k}+G_{j}^{*}\left(p_{k, j}\right) \\
& -\log \frac{\alpha_{4} \epsilon_{k, j}^{4}}{\left(\sqrt{\rho_{k} h_{k, j}}\right)^{4}} \\
& =O\left(\epsilon_{k}\right)+A_{k, j}+\left(\frac{\rho_{k, j}}{4 \sigma_{3}}-8\right) \log \frac{1}{\left|x-p_{k, j}\right|}
\end{aligned}
$$


where $A_{k, j}$ is a constant given by

$$
A_{k, j}=-c_{k}-\log \epsilon_{j}^{4}+G_{j}^{*}\left(P_{k, j}\right)-\log \frac{\alpha_{4}}{\rho_{k}^{2} h_{k, j}^{2}} .
$$

Then by Corollary 2.5, we have $\left|A_{k, j}\right|=O(1)$. This implies that for $x \in \partial B_{\delta_{0}}\left(p_{k, j}\right)$, we have

$$
\eta_{k, j}(x)=A_{k, j}+\left(\frac{\rho_{k, j}}{4 \sigma_{3}}-8\right) \log \frac{1}{\left|x-p_{k, j}\right|}+O\left(\epsilon_{k}\right) .
$$

Moreover, (4.17) holds for partial derivatives of $\eta_{k, j}$ up to the order 3.

Let

$$
\tilde{\eta}_{k, j}(z)=\eta_{k, j}\left(p_{k, j}+\epsilon_{k, j}\left(\rho_{k} h_{k, j}\right)^{-1 / 4} z\right), \quad R=\frac{\delta_{0}}{8 \epsilon_{k, j}} .
$$

The following estimate is the key estimate, whose proof will be given in a separate section:

Estimate C. For any $\tau \in(0,1)$, there exists a constant $C_{\tau}$ such that

$$
\left|\tilde{\eta}_{k, j}(z)\right| \leq C_{\tau}(1+|z|)^{\tau}\left(\epsilon^{2 \tau}+\epsilon^{\tau} \sup _{\frac{R}{2} \leq|z| \leq R}\left|\tilde{\eta}_{k, j}(z)\right|\right) .
$$

Now we have:

Estimate D. For any $\frac{1}{2}<\tau<1$, we have

$$
\left|A_{k, j}\right| \leq C\left(\epsilon+\epsilon^{\tau} \sup _{\frac{R}{2} \leq|z| \leq R}\left|\tilde{\eta}_{k, j}\right|\right) .
$$

Proof. By Green's formula,

$$
\begin{aligned}
u_{k}\left(p_{k, j}\right) & =\log \left(\alpha_{4} \epsilon_{k, j}^{-4}\right)+c_{k} \\
& =\sum_{i=1}^{m} \int_{B_{\delta_{0}}\left(p_{k, l}\right)} \rho_{k} h e^{\tilde{u}_{k}} G_{4}\left(p_{k, j}, y\right) d y+O\left(\epsilon_{k}\right) .
\end{aligned}
$$

For $l \neq j$,

$$
\int_{B_{\delta_{0}}\left(p_{k, l}\right)} \rho_{k} h e^{\tilde{u}_{k}} G_{4}\left(p_{k, j}, y\right) d y=\rho_{k, l} G_{4}\left(p_{k, j}, p_{k, l}\right)+O\left(\epsilon_{k}\right) .
$$

For $l=j$, we have

$$
\begin{aligned}
& \int_{B_{\delta_{0}}\left(p_{k, j}\right)} \rho_{k} h e^{\tilde{u}_{k}} G_{4}\left(p_{k, j}, y\right) d y \\
= & \int_{B_{\delta_{0}}\left(p_{k, j}\right)} \rho_{k} h e^{\tilde{u}_{k}}\left(\frac{1}{4 \sigma_{3}} \log \frac{1}{\left|p_{k, j}-y\right|}+R_{4}\left(p_{k, j}, y\right)\right) d y \\
= & -\frac{1}{4 \sigma_{3}} \rho_{k} \int_{B_{\delta_{0}}\left(p_{k, j}\right)} \rho_{k} h e^{\tilde{u}_{k}} \log \frac{1}{\left|p_{k, j}-y\right|} d y+\rho_{k, j} R_{4}\left(p_{k, j}, p_{k, j}\right)+O\left(\epsilon_{k}\right) .
\end{aligned}
$$


Now we write

$$
h e^{\tilde{u}_{k}}=h e^{v_{k, j}+\eta_{k, j}+\left(G_{j}^{*}(x)-G_{j}^{*}\left(p_{k, j}\right)\right)}=h\left(p_{k, j}\right) e^{v_{k, j}} H\left(x, \eta_{k, j}(x)\right)+h\left(p_{k, j}\right) e^{v_{k, j}}
$$

where

$$
H(x, t)=e^{t+\log h(x)+G_{j}^{*}(x)-\log h\left(p_{k, j}\right)-G_{j}^{*}\left(p_{k, j}\right)}-1 .
$$

Let $z$ and $z_{k, j}$ satisfy

$$
x=p_{k, j}+\epsilon_{k, j}\left(\rho_{k} h_{k, j}\right)^{-1 / 4} z, \quad q_{k, j}=p_{k, j}+\epsilon_{k, j}\left(\rho_{k} h_{k, j}\right)^{-1 / 4} z_{k, j} .
$$

Then we have

$$
\begin{aligned}
& \rho_{k} \int_{B_{\delta}\left(P_{k, j}\right)} h e^{\tilde{u}_{k}} \log \left|p_{k, j}-y\right| \\
& =\rho_{k} \int_{B_{\delta_{0}}\left(p_{k, j}\right)} h e^{\tilde{u}_{k}} \log \epsilon_{k, j}\left(\rho_{k} h_{k, j}\right)^{-1 / 4}+\rho_{k} \int_{B_{R}(0)} h e^{\tilde{u}_{k}} \log |z| \\
& =\rho_{k, j} \log \left(\epsilon_{k, j}\left(\rho_{k} h_{k, j}\right)^{-1 / 4}\right) \\
& \quad+\rho_{k} \int_{B_{R}(0)} \frac{\alpha_{4}}{\left(1+\left|z-z_{k, j}\right|^{2}\right)^{4}}\left(1+H\left(x, \eta_{k, j}\right)\right) \log |z| \\
& =\rho_{k, j} \log \left(\epsilon_{k, j}\left(\rho_{k} h_{k, j}\right)^{-1 / 4}\right) \\
& \quad+\rho_{k} \int_{B_{R}(0)} \frac{\alpha_{4}}{\left(1+\left|z-z_{k, j}\right|^{2}\right)^{4}} H\left(x, \eta_{k, j}\right) \log |z|+O\left(\epsilon_{k}\right)
\end{aligned}
$$

where we have used

$$
\left|z_{k, j}\right|=O\left(\epsilon_{k}\right), \int_{0}^{\infty}\left(1+r^{2}\right)^{-4} r^{3} \log r d r=0 .
$$

The last term in (4.23) can be estimated by

$$
\begin{gathered}
\int_{B_{R}(0)} \frac{\alpha_{4}}{\left(1+\left|z-z_{k, j}\right|^{2}\right)^{4}} H(x, 0) \log |z|+O\left(\epsilon_{k}^{2 \tau}+\epsilon_{k}^{\tau} \sup _{\frac{R}{2} \leq|z| \leq R}\left|\tilde{\eta}_{k, j}(z)\right|\right) \\
=O\left(\epsilon_{k}+\epsilon_{k}^{\tau} \sup _{\frac{R}{2} \leq|z| \leq R}\left|\tilde{\eta}_{k, j}(z)\right|\right) .
\end{gathered}
$$

Combining all together, we obtain Estimate D (by choosing a larger $\tau>\frac{1}{2}$ ). 
Estimate D implies:

Estimate E. On $B_{\frac{\delta_{0}}{2}}\left(p_{k, j}\right)$

$$
\eta_{k, j}(x)=\left(\frac{\rho_{k, j}}{4 \sigma_{3}}-8\right) \log \frac{1}{\left|x-p_{k, j}\right|}+O\left(\epsilon+\epsilon^{\tau} \sup _{\frac{R}{2} \leq|z| \leq R}\left|\tilde{\eta}_{k, j}(z)\right|\right) .
$$

From (4.25), we also have

$$
\sup _{\frac{R}{2} \leq|z| \leq R}\left|\tilde{\eta}_{k, j}\right| \leq C\left|\rho_{k, j}-32 \sigma_{3}\right|+C \epsilon_{k, j}
$$

Hence Estimate $\mathrm{C}$ can be refined as

$$
\left|\tilde{\eta}_{k, j}(z)\right| \leq C(1+|z|)^{\tau}\left(\epsilon_{k}^{2 \tau}+\epsilon_{k}^{\tau}\left|\rho_{k, j}-32 \sigma_{3}\right|\right) .
$$

On the other hand, $\left|\rho_{k, j}-32 \sigma_{3}\right|$ can also be estimated by a quantity related to $\eta_{k, j}$.

\section{Estimate F.}

$$
\rho_{k, j}-32 \sigma_{3}=\int_{\partial B_{\delta_{0}}\left(p_{k, j}\right)} \frac{\partial \Delta \eta_{k, j}}{\partial v} d x+o\left(\epsilon_{k}^{2}\right)
$$

Proof.

$$
\begin{aligned}
\rho_{k, j} & =\rho_{k} \int_{B_{\delta}\left(p_{k, j}\right)} h e^{\tilde{u}_{k}}=\int_{\partial B_{\delta_{0}}\left(p_{k, j}\right)} \frac{\partial \Delta u_{k}}{\partial v} \\
& =\int_{\partial B_{\delta_{0}}\left(p_{k, j}\right)} \frac{\partial \Delta v_{k, j}}{\partial v}+\int_{\partial B_{\delta_{0}}\left(p_{k, j}\right)} \frac{\partial \Delta \eta_{k, j}}{\partial v} \\
& =32 \sigma_{3}+\int_{\partial B_{\delta_{0}}\left(p_{k, j}\right)} \frac{\partial \Delta \eta_{k, j}}{\partial v}+O\left(\epsilon_{k}^{3}\right)
\end{aligned}
$$

since

$$
\frac{\partial \Delta v_{k, j}}{\partial v}=32 \frac{1}{r^{3}}+O\left(\frac{1}{r^{7}}\right)
$$

It remains to compute $\int_{\partial B_{\delta_{0}}\left(p_{k, j}\right)} \frac{\partial \Delta \eta_{k, j}}{\partial v}$. 
Note that $\eta_{k, j}$ satisfies

$$
\Delta^{2} \eta_{k, j}=\rho_{k} h_{k, j} e^{v_{k, j}} \eta_{k, j}+\rho_{k} h_{k, j} e^{v_{k, j}}\left(H\left(x, \eta_{k, j}\right)-\eta_{k, j}\right) .
$$

Let

$$
\Psi(x)=\frac{\epsilon_{k, j}^{2}-\sqrt{\rho_{k} h_{k, j}}\left|x-q_{k, j}\right|^{2}}{\epsilon_{k, j}^{2}+\sqrt{\rho_{k} h_{k, j}}\left|x-q_{k, j}\right|^{2}} .
$$

Then it is easy to see that $\Psi(x)$ satisfies

$$
\Delta^{2} \Psi(x)=\rho_{k} h_{k, j} e^{v_{k, j}(x)} \Psi(x) .
$$

Using (4.29) and (4.30), we obtain

$$
\begin{aligned}
& \int_{B_{\delta_{0}}\left(p_{k, j}\right)}\left[\Delta^{2} \eta_{k, j} \Psi(x)-\Delta^{2} \Psi(x) \eta_{k, j}(x)\right] \\
& =\int_{B_{\delta_{0}}\left(p_{k, j}\right)} \rho_{k} h_{k, j} e^{v_{k, j}}\left[H\left(x, \eta_{k, j}\right)-\eta_{k, j}\right] \Psi(x) .
\end{aligned}
$$

The left-hand-side of (4.31) equals

$$
\begin{aligned}
= & \int_{\partial B_{\delta_{0}}\left(p_{k, j}\right)}\left[\frac{\partial \Delta \eta_{k, j}}{\partial v} \Psi(x)-\Delta \eta_{k, j} \frac{\partial \Psi}{\partial v}+\Delta \Psi \frac{\partial \eta_{k, j}}{\partial v}-\eta_{k, j} \frac{\partial \Delta \Psi}{\partial v}\right] \\
= & \int_{\partial B_{\delta_{0}}\left(p_{k, j}\right)} \frac{\partial \Delta \eta_{k, j}}{\partial v}(\Psi(x)+1) \\
& -\int_{\partial B_{\delta_{0}}\left(p_{k, j}\right)} \frac{\partial \Delta \eta_{k, j}}{\partial v}+O\left(\epsilon_{k, j}^{2}\left|\rho_{k, j}-32 \sigma_{3}\right|+\epsilon_{k, j}^{3}\right) \\
= & \int_{\partial B_{\delta_{0}}\left(p_{k, j}\right)} \frac{\partial \Delta \eta_{k, j}}{\partial v} \frac{2 \epsilon_{k, j}^{2}}{\epsilon_{k, j}^{2}+\sqrt{\rho_{k} h_{k, j}} \delta_{0}^{2}+o\left(\epsilon_{k, j}^{2}\right)} \\
& -\int_{\partial B_{\delta_{0}}\left(p_{k, j}\right)} \frac{\partial \Delta \eta_{k, j}}{\partial v}+O\left(\epsilon_{k, j}^{2}\left|\rho_{k, j}-32 \sigma_{3}\right|+\epsilon_{k, j}^{3}\right) \\
= & -\int_{\partial B_{\delta_{0}}\left(p_{k, j}\right)} \frac{\partial \Delta \eta_{k, j}}{\partial v}+o\left(\epsilon_{k, j}^{2}\right) .
\end{aligned}
$$


The right-hand-side of (4.31) equals

$$
\begin{aligned}
=\frac{1}{\sqrt{\rho_{k} h_{k, j}}} \int_{\mathbb{R}^{4}}\left(\frac{1}{\left(1+\left|z-z_{k, j}\right|^{2}\right)^{4}}\right)\left[\epsilon_{k, j}^{2} \sum_{l, m} \frac{\partial^{2}}{\partial x_{l} \partial x_{m}}\left[G_{j}^{*}+\log h\right] z_{l} z_{m}\right. \\
\left.+O\left(\left|\eta_{k, j}\right|^{2}\right)\right] \frac{1-\left|z-z_{k, j}\right|^{2}}{1+\left|z-z_{k, j}\right|^{2}} d z \\
=\frac{1}{\sqrt{\rho_{k} h_{k, j}}} \int_{\mathbb{R}^{4}}\left(\frac{1}{\left(1+|z|^{2}\right)^{4}}\right)\left[\epsilon_{k, j}^{2} \sum_{l, m} \frac{\partial^{2}}{\partial x_{l} \partial x_{m}}\left[G_{j}^{*}+\log h\right]\left(z_{l}+z_{k, l}\right)\left(z_{m}+z_{k, m}\right)\right. \\
\left.\quad+O\left(\epsilon_{k}^{4 \tau}\right)\right] \frac{1-|z|^{2}}{1+|z|^{2}} d z \\
=\frac{1}{\sqrt{\rho_{k} h\left(p_{k, j}\right)}} \epsilon_{k, j}^{2} \Delta\left(G_{j}^{*}+\log h\right)\left(p_{k, j}\right) \frac{1}{4} \int_{\mathbb{R}^{4}} \frac{|z|^{2}}{\left(1+|z|^{2}\right)^{4}} \frac{1-|z|^{2}}{1+|z|^{2}} d z+o\left(\epsilon_{k, j}^{2}\right)
\end{aligned}
$$

where

$$
\frac{1}{4} \int_{\mathbb{R}^{4}} \frac{|z|^{2}}{\left(1+|z|^{2}\right)^{4}} \frac{1-|z|^{2}}{1+|z|^{2}} d z=\frac{1}{4} \int_{\mathbb{R}^{4}} \frac{|z|^{2}-1}{\left(1+|z|^{2}\right)^{4}} \frac{1-|z|^{2}}{1+|z|^{2}} d z<0 .
$$

Combining Estimate F and (4.32), we obtain

$$
\rho_{k, j}-32 \sigma_{4}=c_{0} \frac{1}{\sqrt{h\left(p_{k, j}\right)}} \epsilon_{k, j}^{2} \Delta\left(G_{j}^{*}\left(p_{k, j}\right)+\log h\left(p_{k, j}\right)\right)+o\left(\epsilon_{k, j}^{2}\right)
$$

where $c_{0}>0$.

Finally, summing up the estimates in (4.34), we obtain Theorem 1.2.

\section{Proof of Estimate $\mathbf{C}$}

In this section, we prove Estimate C. Our proof is different from [7] and is simpler.

Let $\tau \in(0,1)$ be a fixed positive number. We begin with the following simple but important lemma:

Lemma 5.1. Let $u$ satisfy

$$
\Delta^{2} u=f(y) \text { in } B_{R}(0), \quad u=\Delta u=0 \text { on } \partial B_{R}(0)
$$

Then for $R$ large we have

$$
\left\|<y>^{-\tau}(u-u(0))\right\|_{L^{\infty}\left(B_{\frac{R}{2}}(0)\right)} \leq C\left\|<y>^{4-\tau} f(y)\right\|_{L^{\infty}\left(B_{R}(0)\right)} .
$$


Proof. Assume that $\left\|<y>^{4-\tau} f(y)\right\|_{L^{\infty}\left(B_{R}(0)\right)}=1$.

By the Green's representation formula,

$$
u(y)-u(0)=\int_{B_{R}(0)}\left[\frac{1}{4 \sigma_{3}} \log \frac{|z|}{|y-z|}+\hat{R}_{4}\left(\frac{y}{R}, \frac{z}{R}\right)-\hat{R}_{4}\left(0, \frac{z}{R}\right)\right] f(z) d z
$$

where $\hat{R}_{4}$ is the regular part of the Green's function of $\Delta^{2}$ in $B_{1}(0)$ with Navier boundary condition.

Note that

$$
\begin{gathered}
\int_{B_{R}(0)}\left|\hat{R}_{4}\left(\frac{y}{R}, \frac{z}{R}\right)-\hat{R}_{4}\left(0, \frac{z}{R}\right)\right||f(z)| d z \leq C \frac{1}{R^{\tau}} \int_{B_{R}(0)}<y>^{\tau}|f(z)| d z \\
\leq C<y>^{\tau} .
\end{gathered}
$$

So we only need to consider the first term on the right hand side of (5.2). To this end, we decompose

$$
\int_{B_{R}(0)} \log \frac{|z|}{|y-z|} f(z) d z=\left(\int_{|z| \leq \frac{1}{2}|y|}+\int_{\frac{1}{2}|y| \leq|z| \leq 2|y|}+\int_{|z| \geq 2|y|}\right) \log \frac{|z|}{|y-z|} f(z) d z .
$$

The last integral can be controlled by

$$
\left|\int_{|z| \geq 2|y|} \log \frac{|z|}{|y-z|} f(z) d z\right| \leq C\left|\int_{|z| \geq 2|y|} \frac{|y|}{|z|}\right| f(z) \mid d z \leq C<y>^{\tau} .
$$

For the first integral, we have

$$
\begin{aligned}
|y-z| \geq|y|-|z| & \geq \frac{1}{2}|y| \geq|z| \\
\left|\int_{|z| \leq \frac{1}{2}|y|} \log \frac{|z|}{|y-z|} f(z) d z\right| & \leq \int_{|z| \leq \frac{1}{2}|y|} \log \frac{|y-z|}{|z|}<z>^{\tau-4} d z \\
& \leq C<y>^{\tau} .
\end{aligned}
$$

It remains to compute the last integral

$$
\begin{aligned}
\int_{\frac{1}{2}|y| \leq|z| \leq 2|y|} \log \frac{|z|}{|y-z|} f(z) d z & =|y|^{4} \int_{\frac{1}{2} \leq|\tilde{z}| \leq 2} \log \frac{|\tilde{z}|}{\left|e_{1}-\tilde{z}\right|} f(|y| \tilde{z}) d \tilde{z} \\
& \leq|y|^{4} \int_{\frac{1}{2} \leq|\tilde{z}| \leq 2}\left|\log \frac{|\tilde{z}|}{\left|e_{1}-\tilde{z}\right|}\right|<y>^{\tau-4}<\tilde{z}>^{\tau-4} d \tilde{z} \\
& \leq C<y>^{\tau} .
\end{aligned}
$$


Let us go back to the equation for $\tilde{\eta}$ :

$$
\begin{gathered}
\Delta^{2} \tilde{\eta}=e^{U}\left(e^{\tilde{\eta}}-1\right)+O\left(\epsilon_{k, j}^{2}<y>^{-6}\right) \text { in } B_{\frac{\delta_{0}}{\epsilon_{k, j}}}(0) \\
\tilde{\eta}=O(1), \Delta \tilde{\eta}=O\left(\epsilon_{k, j}^{2}\right) \text { on } \partial B_{\frac{\delta_{0}}{\epsilon_{k, j}}}(0) .
\end{gathered}
$$

Let $\hat{\eta}_{k, j}=\tilde{\eta}_{k, j} \chi$. Then we have $\hat{\eta}_{k, j}$ satisfies

$$
\begin{aligned}
\Delta^{2} \hat{\eta}_{k, j} & =e^{U} \frac{\left(e^{\tilde{\eta}_{k, j}}-1\right)}{\tilde{\eta}_{k, j}} \hat{\eta}_{k, j}+O\left(\epsilon_{k, j}^{2}<y>^{-6}\right) \text { in } B_{\frac{\delta_{0}}{\epsilon_{k, j}}}(0), \\
\hat{\eta}_{k, j} & =\Delta \hat{\eta}_{k, j}=0 \text { on } \partial B_{\frac{\delta_{0}}{\epsilon_{k, j}}}(0) .
\end{aligned}
$$

We claim that

$$
\left\|<y>^{-\tau} \hat{\eta}_{k, j}\right\|_{L^{\infty}\left(B \frac{\delta}{2 \epsilon_{k, j}}(0)\right)} \leq C \epsilon_{k, j}^{\tau} .
$$

Estimate C follows from (5.4).

Suppose not. Then $\left.\epsilon_{k, j}^{-\tau}\left\|<y>^{-\tau} \hat{\eta}_{k, j}\right\|_{L^{\infty}\left(B \frac{\delta_{0}}{2 \epsilon_{k, j}}\right.}(0)\right) \rightarrow+\infty$. Let $\bar{\eta}_{k, j}=$ $\frac{\hat{\eta}_{k, j}}{\left\|<y>^{-\tau} \hat{\eta}_{k, j}\right\|_{L^{\infty}(B} \frac{\delta}{2 \epsilon_{k, j}}}{ }^{(0))}$. Then $\bar{\eta}_{k, j}$ satisfies

$$
\begin{aligned}
\Delta^{2} \bar{\eta}_{k, j} & =e^{U} \frac{\left(e^{\tilde{\eta}_{k, j}}-1\right)}{\tilde{\eta}_{k, j}} \bar{\eta}_{k, j}+o\left(<y>^{\tau-4}\right) \text { in } B_{\frac{\delta}{\epsilon_{k, j}}}(0), \\
\bar{\eta}_{k, j} & =\Delta \bar{\eta}_{k, j}=O \text { on } \partial B_{\frac{\delta}{\epsilon_{k, j}}}(0)
\end{aligned}
$$

where $\bar{\eta}_{k, j}(0)=0$.

We claim that $\bar{\eta}_{k, j} \rightarrow 0$ in $C_{\mathrm{loc}}^{1}\left(\mathbb{R}^{4}\right)$. In fact, by standard elliptic regularity theory, $\bar{\eta}_{k, j} \rightarrow \eta_{0}$, where $\eta_{0}$ satisfies

$$
\Delta^{2} \eta_{0}=e^{U} \eta_{0}, \eta_{0}(0)=\nabla \eta_{0}(0)=0,\left|\eta_{0}(y)\right| \leq C<y>^{\tau} .
$$

By Lemma 2.6, $\eta_{0}=\sum_{j=0}^{4} c_{j} \psi_{j}$ for some constants $c_{j}, j=0,1, \ldots, 4$. Using the assumption $\eta_{0}(0)=\nabla \eta_{0}(0)=0$, we deduce that $c_{j}=0$ and hence $\eta_{0} \equiv 0$.

So $\bar{\eta}_{k, j} \rightarrow 0$ in $C_{\text {loc }}^{1}\left(\mathbb{R}^{4}\right)$. Now we consider

$$
\begin{aligned}
& \left\|<y>^{4-\tau} e^{U} \frac{\left(e^{\tilde{\eta}_{k, j}}-1\right)}{\tilde{\eta}_{k, j}} \bar{\eta}_{k, j}\right\|_{L^{\infty}\left(B_{\frac{\delta_{0}}{\epsilon_{k, j}}}(0)\right)}=o(1) . \\
& \leq C\left\|<y>^{4-\tau}<y>^{-8} \bar{\eta}_{k, j}\right\|_{L^{\infty}\left(B_{\frac{\delta}{\epsilon_{k, j}}}(0)\right)}=o(
\end{aligned}
$$


By Lemma 5.1, we conclude

$$
\begin{aligned}
& \left.\left\|<y>^{-\tau} \bar{\eta}_{k, j}\right\|_{L^{\infty}\left(B \frac{\delta}{2 \epsilon_{k, j}}\right.}(0)\right) \\
& \leq C\left\|<y>^{-\tau-4} e^{U}\left(e^{\tilde{\eta}_{k, j}}-1\right) \tilde{\eta}_{k, j}^{-1} \bar{\eta}_{k, j}\right\|_{L^{\infty}\left(B \frac{\delta}{2 \epsilon_{k, j}}(0)\right)}+o(1)=o(1)
\end{aligned}
$$

which contradicts to the assumption that $\left\|<y>^{-\tau} \bar{\eta}_{k, j}\right\|_{L^{\infty}\left(B_{\frac{\delta}{2 \epsilon_{k, j}}}(0)\right)}=1$.

\section{Appendix A: Proof of Lemma 2.1}

In this appendix, we prove Lemma 2.1. We follow the ideas in Section 2 of our previous paper [16].

Let $u_{k}$ be a sequence of solutions of

$$
\begin{cases}\Delta^{2} u_{k}=\mu_{k} h(x) e^{u_{k}}, & \text { in } \Omega \\ u_{k}=0 & \text { on } \partial \Omega\end{cases}
$$

and $u_{k}$ blows up at $\left\{q_{1}, \ldots, q_{n}\right\}$, where $\mu_{k}=\frac{\rho_{k}}{\int_{\Omega} h(x) e^{u_{k}}} \rightarrow 0$.

In our previous paper [16], we have shown that $u_{k} \rightarrow P(x)$ in $C^{4}\left(\bar{\Omega} \backslash\left\{p_{1}, \ldots, p_{m}\right\}\right)$. It is not difficult to show that

$$
\lim _{x \rightarrow p_{j}} P(x)=\lim _{x \rightarrow p_{j}}(-\Delta P(x))=+\infty, j=1, \ldots, m
$$

Claim 5.1. $p_{j} \in \Omega$ for $j=1,2, \ldots, m$.

We prove it by contradiction. Assume $p_{1}=0 \in \partial \Omega$ and $e_{1}=(1,0, \ldots, 0)$ is the outernormal of $\partial \Omega$ at zero. (See [16, Figure 2.1].)

Let $N=B(0, r) \cap \Omega$ and $\varphi$ be a solution of

$$
\begin{cases}\Delta^{2} \varphi=0 & \text { in } \Omega, \\ \Delta \varphi<0 \text { and } \varphi>0 & \text { in } \Omega, \\ \varphi=0 & \text { on } B(0, r) \cap \partial \Omega, \\ \Delta \varphi=0 & \text { on } B(0, r) \cap \partial \Omega .\end{cases}
$$

We now choose $C$ is a large number so that

$$
h e^{C \varphi(x)} \text { is decreasing in } x_{1} \text { for } x \in N \text {. }
$$

$N$ can be chosen so small such that

$$
P(x)-C \varphi(x) \geq 0 \text { and } \Delta P(x)-C \Delta \varphi(x) \leq 0
$$


for $x \in \partial N$. Therefore by the maximum principle, for large $k$,

$$
u_{k}-C \varphi(x)>0 \text { and } \Delta u_{k}(x)-C \Delta \varphi(x)<0
$$

for $x \in \partial N \cap \Omega$. Set $w_{k}=u_{k}-C \varphi(x)$. Then

$$
\begin{aligned}
\Delta^{2} w_{k} & =\mu_{k}\left(h e^{C \varphi}\right) e^{w_{k}} \text { in } N \\
w_{k} & =\Delta w_{k}=0 \text { for } x \in \partial \Omega \cap N .
\end{aligned}
$$

Then we can use the local version of the moving planes as before to show $w_{k}(x)$ is decreasing in $x_{1}$, which yields a contradiction to the assumption 0 is a blowup point.

\section{Appendix B: Proof of Lemma 2.6}

In this section, we prove the nondegeneracy Lemma 2.6

Let $U=\log \frac{\alpha_{4}}{\left(1+|y|^{2}\right)^{4}}$ be a solution of $\Delta^{2} u=e^{u}$. In this section, we study the following eigenvalue problem

$$
\Delta^{2} \phi=e^{U} \phi,|\phi| \leq C<y>^{\tau}
$$

for some $\tau \in(0,1)$.

Since $\psi_{j}, j=0, \ldots, 4$ satisfies (5.6), we may assume that $\phi$ satisfies $\int_{\mathbb{R}^{4}} e^{U} \psi_{j} \phi=$ $0, j=0,1, \ldots, 4$. Our goal is then to show that $\phi \equiv 0$.

Let $\phi_{0}=\frac{1}{4 \sigma_{3}} \int_{\mathbb{R}^{4}} \log \frac{1}{|y-z|} e^{U} \phi d z$. Then we have $\Delta^{2}\left(\phi-\phi_{0}\right)=0$. Since $\left|\phi_{0}(y)\right| \leq C \log (2+|y|)$, we have $\phi-\phi_{0}=C$. Thus,

$$
\phi(y)=\frac{1}{4 \sigma_{3}} \int_{\mathbb{R}^{4}} \log \frac{1}{|y-z|} e^{U} \phi d z+C .
$$

We first prove:

Lemma 5.1. Let $\phi$ satisfy (5.6). Then $|\phi| \leq C$ and there holds

$$
\int_{\mathbb{R}^{4}} e^{U} \phi=0, \int_{\mathbb{R}^{4}} e^{U} \psi_{j} \phi=0, j=0,1, \ldots, 4 .
$$

Proof. From (5.7), we see that

$$
\phi=\ln |y|\left(\frac{1}{4 \sigma_{3}} \int_{\mathbb{R}^{4}} e^{U} \phi\right)+O(1), \text { for }|y| \geq 1
$$

and (5.9) holds for $D^{\alpha} \phi,|\alpha| \leq 3$.

We decompose

$$
\phi=\phi_{0}(r)+\phi^{\prime}
$$


where $\phi_{0}(r)=\frac{1}{\left|S^{3}\right|} \int_{S^{3}} \phi d \sigma$. Then it is easy to see that both $\phi_{0}$ and $\phi^{\prime}$ satisfy (5.6). Since $U$ is radially symmetric, by (5.9), it is easy to see that $\phi^{\prime}$ is bounded and satisfies (5.8). Therefore, to prove the Lemma, it is enough to assume that $\phi=\phi(r)$ is radially symmetric. Now multiplying (5.6) by $\psi_{0}(r)=\frac{1-r^{2}}{1+r^{2}}$ and integrating over $B_{r}(0)$, we obtain

$$
0=\int_{\partial B_{r}(0)} \psi_{0}(r) \frac{\partial \Delta \phi}{\partial r}-\phi(r) \frac{\partial \Delta \psi_{0}}{\partial r}
$$

and hence for $r$ large, we have

$$
\frac{\partial \Delta \phi}{\partial r}=-\phi(r) \frac{\partial \Delta \psi_{0}}{\partial r}=O\left(\frac{\ln r}{r^{5}}\right) .
$$

From (5.9) and (5.11), we see that necessarily, $\int_{\mathbb{R}^{4}} e^{U} \phi=0$. The lemma is thus proved.

Let $\Pi$ be the stereographic projection of the sphere $S^{4}$ onto $\mathbb{R}^{4}$ with respect to the North Pole. Namely,

$$
\forall x=\left(x_{1}, \ldots, x_{5}\right) \in S^{4}, y_{i}=\frac{x_{i}}{1-x_{5}}, i=1, \ldots, 4, \Pi(x)=y=\left(y_{1}, \ldots, y_{4}\right) .
$$

For a bounded function $\phi(y)$ defined on $\mathbb{R}^{4}$, one can define a function $\psi$ on $S^{4}$ by $\psi(x)=\phi(y), y=\Pi(x)$. Then it is easy to see that

$$
\begin{aligned}
\int_{S^{4}} \psi(\sigma) d \sigma & =\int_{\mathbb{R}^{4}} \phi(y) e^{U} d y \\
\int_{S^{4}}\left(\mathbb{P}_{4} \psi, \psi\right) & =\int_{\mathbb{R}^{4}}(\Delta \phi)^{2}
\end{aligned}
$$

where $\mathbb{P}_{4}=(-\Delta)(-\Delta+2)$ is the Paneitz operator on $S^{4}$.

Transforming the identities (5.8) to $\psi$, we have that $\psi$ satisfies

$$
\int_{S^{4}} \psi d \sigma=\int_{S^{4}} \psi x_{i} d \sigma=0, i=1, \ldots, 5 .
$$

Note that the operator $-\Delta_{S^{n}}$ is known to have eigenvalues $\lambda_{j}$ with multiplicity $n_{j}$ and eigenfunctions $u_{j}$ as follows:

$$
\begin{aligned}
& \lambda_{0}=0, n_{0}=1, u_{0}=1 \\
& \lambda_{0}=n, n_{1}=n+1, u_{1, i}=\sigma_{i}, i=1, \ldots, n+1 \\
& \lambda_{k}=k(n+k-1), n_{k}=\frac{(n+k-2) !(n+2 k-1) !}{k !(n-1) !}, u_{k, i} .
\end{aligned}
$$


Thus $\mathbb{P}_{4}=(-\Delta)(-\Delta+2)$ also has eigenvalues and eigenfunctions

$$
\begin{aligned}
& \mu_{0}=0, u_{0}=1, \\
& \mu_{1}=24, u_{1, i}=x_{i}, i=1, \ldots, 5 \\
& \mu_{2}>24 .
\end{aligned}
$$

From (5.6) and (5.14), we derive that

$$
\int_{S^{4}}\left(\mathbb{P}_{4} \psi, \psi\right)=24 \int_{\mathbb{R}^{4}} e^{U} \phi^{2}=24 \int_{S^{4}} \psi^{2} d \sigma \geq \mu_{2} \int_{S^{4}} \psi^{2}
$$

which implies $\psi=0$ and so $\phi=0$.

This proves Lemma 2.6.

\section{References}

[1] Adimurthi, F. Robert and M. Struwe, Concentration phenomena for Liuville equations in dimension four, J. Eur. Math. Soc. 8 (2006), 171-180.

[2] H. BREZIS and F. MERLE, Uniform estimates and blow-up behavior for solutions of $-\Delta u=V(x) e^{u} i$ ntwo dimensions, Comm. Partial Differential Equation 16 (1991), 12231254.

[3] S. Baraket, M. Dammak, T. OUni, and F. PaCard, Singular limits for 4-dimensional semilinear elliptic problems with exponential nonlinearity, Ann. Inst. H. Poincaré Anal. Non Linéaire 24 (2007), 875-895.

[4] D. Bartolucci, C.-C. Chen, C.-S. Lin and G. Tarantello, Profile of blow-up solutions to mean field equations with singular data, Comm. Partial Differential Equations 29 (2004), 1241-1265.

[5] H. BREZIS, Y. Y. LI and I. SHAFRIR, A sup + inf inequality for some nonlinear elliptic equations involving exponential nonlinearities, J. Funct. Anal. 115 (1993), 344-358.

[6] S-Y A. CHANG and P. C. YANG, Extremal metrics of zeta function determinants on 4manifolds, Ann. of Math. 142 (1995), 171-212.

[7] C. C CHEN and C. S. Lin, Sharp estimates for solutions of multi-bubbles in compact Riemann surface, Comm. Pure Appl. Math. 55 (2002), 728-771.

[8] C. C. CHEN and C. S. Lin, Topological degree for a mean field equation on Riemann surfaces, Comm. Pure Appl. Math. 56 (2003), 1667-1727.

[9] O. DRUET and F. ROBERT, Bubbling phenomena for fourth-order four-dimensional PDEs with exponential growth, Proc. Amer. Math. Soc. 134 (2006), 897-908.

[10] E. HeBeY and F. RoberT, Coercivity and Struwe's compactness for Paneitz type operators with constant coefficients, Cal. Var. Partial Differential Equations 13 (2001), 491-517.

[11] E. HeBey, F. ROBERT and Y. WEN, Compactness and global estimates for a fourth order equation with critical Sobolev growth arising from conformal geometry, Comm. Contemp. Math. 8 (2006), 9-65.

[12] Y. Y. LI, Harnack inequality: the method of moving planes, Comm. Math. Phys. 200 (1999), 421-444.

[13] Y. Y. LI and I. SHAFRIR, Blow-up analysis for solutions of $-\Delta u=V e^{u}$ in dimension two, Indiana Univ. Math. J. 43 (1994), 1255-1270.

[14] C. S. LIN, Locating the peaks of solutions to a Neumann problem via the maximum principle, I: The Neumann problem, Comm. Pure Appl. Math. 56 (2001), 1065-1095. 
[15] C. S. LIN, A classification of solutions of a conformally invariant fourth order equation in $R^{4}$, Comment. Math. Helv. 73 (1998), 206-231.

[16] C. S. LIN and J.-C. WEI, Locating the peaks of solutions via the maximum principle. II. A local version of the method of moving planes, Comm. Pure Appl. Math. 56 (2003), 784-809.

[17] C. S. LIN, L.-P. WANG and J.-C. WEI, Topological degree for 4-dimensional mean field equations, submitted.

[18] L. MA and J. WEI, Convergence for a Liouville equation, Comment. Math. Helv. 76 (2001), 506-514.

[19] A. MALCHIODI, Compactness of solutions to some geometric fourth-order equations, J. Reine Angew. Math. 594 (2006), 137-174.

[20] A. MALCHIODI, Morse theory and a scalar field equation on compact surfaces, preprint.

[21] K. NAGASAKI and T. SuzUKI, Asymptotic analysis for two-dimensional elliptic eigenvalue problems with exponentially dominated nonlinearity, Asymptot. Anal. 3 (1990), $173-188$.

[22] J. C. WEI and X. W. XU, Classification of solutions of higher order conformally invariant equations, Math. Ann. 313 (1999), 207-228.

[23] J. WEI, Asymptotic behavior of a nonlinear fourth order eigenvalue problem, Comm. Partial Differential Equations 21 (1996), 1451-1467.

Department of Mathematics

National Taiwan University

Taipei, Taiwan

cslin@math.ntu.edu.tw

Department of Mathematics

The Chinese University of Hong Kong

Shatin, Hong Kong

wei@math.cuhk.edu.hk 